\title{
Vorwort zu Band 3/2
}

Materialien und Materialsysteme bilden das Fundament der Nanotechnologie und sind in ihren unterschiedlichen Ausprägungen Gegenstand der Nanostrukturforschung. Materialien und Systeme können insbesondere gleichsam die Materialisation völlig neuartiger Phänomene sein, wobei hier häufig die materielle Voraussetzung im nanostrukturierten Aufbau der entsprechenden Materialien und Systeme begründet liegt. Diesem Sachverhalt widmet sich in der Hauptsache Band 3 dieser Buchreihe. Wiederum sind viele der vorgestellten Materialien und Systeme quasi funktionelle Manifestationen vieler in Band 1 der Buchreihe behandelter Grundlagen und komplettieren die bereits in Band 2 behandelten Materialien und Systeme. Auch wenn die Bezüge zwischen allen Bänden der vorliegenden Lehrbuchreihe eng sind und Bezüge auch häufig explizit vermerkt werden, so ist ebenfalls Band 3/2 so konzipiert, dass er den enthaltenen Stoff in einer Weise vermittelt, dass ein detailliertes Studium der Bände 1 und 2 nicht zwingend vorausgesetzt wird. Materialien, Systeme und Methoden werden also umfassend erklärend und mit einem optionalen Bezug $\mathrm{zu}$ bereits behandelten Sachverhalten dargestellt. Wiederum werden theoretische, experimentelle und technische Aspekte gleichgewichtig und mit deutlichem Bezug zu allerneuesten Forschungsergebnissen behandelt.

Die theoretische Beschreibung von Nanostrukturen erfordert spezielle Konzepte der Theoriebildung. Im Allgemeinen müssen sowohl räumlich als auch zeitlich unterschiedliche Skalen in adäquater Weise miteinander kombiniert werden. Dies erfordert angemessene und in Bezug auf ihre Realitätsnähe und Genauigkeit wohlverstandene Näherungen. Diese Näherungen sind in der Regel Voraussetzung dafür, dass verwendete numerische Verfahren überhaupt konvergieren. Insbesondere die Beschreibung der Dynamik von Nanostrukturen erweist sich zuweilen als eine außerordentlich komplexe Aufgabenstellung. Im vorliegenden Band werden verschiedene Strategien zur Simulation von Nanostrukturen im Hinblick auf ihren skalenübergreifenden Charakter, die Effizienz von Algorithmen und im Hinblick auf die Präzision und das Ausmaß der Vorhersagbarkeit von Eigenschaften eingehend behandelt. Beispiele mit Querschnittsbedeutung umfassen Tight Binding-Ansätze, Dichtefunktionaltheorie, Monte Carlo-Simulationen und Molekulardynamiksimulationen.

Band 3/2 von Nanostrukturforschung und Nanotechnologie behandelt auch für das Gebiet wichtige experimentelle Methoden dezidiert. $\mathrm{Zu}$ den wichtigsten analytischen Methoden in den Nanowissenschaften, aber auch bei technologischen Anwendungen gehören sicherlich die Rastersondenverfahren, die zuweilen als die Wegbereiter der Nanotechnologie schlechthin angesehen werden. Zunächst werden in diesem Kontext die apparativen Gemeinsamkeiten aller Rastersondenverfahren dargestellt, um dann die drei wichtigsten Vertreter, die Rastertunnelmikroskopie, die Rasterkraftmikroskopie und die optische Rasternahfeldmikroskopie im Detail zu behandeln. 
Die Rastertunnelmikroskopie hat sich als unschätzbar wertvolle Methode bei der Analyse elektronischer Eigenschaften auf Nanometerskala erwiesen, und in den letzten nahezu vierzig Jahren wurden zahllose bahnbrechende Ergebnisse, diverseste Anwendungsmöglichkeiten und viele spezielle Betriebsmodi vorgestellt. Die Diskussion umfasst neben Standardanwendungen der Rastertunnelmikroskopie und Rastertunnelspektroskopie auch speziellere Anwendungen wie die spinpolarisierte Tunnelmikroskopie oder die atomare Manipulation mit dem Tunnelmikroskop.

Da die Rasterkraftmikroskopie nicht die elektrische Leitfähigkeit der Proben voraussetzt, bietet sie a priori ein noch weiteres Anwendungsfeld als die Rastertunnelmikroskopie. Dieses Anwendungsfeld umfasst die Charakterisierung von KraftAbstands-Verläufen, die Abbildung lokaler Ladungsvariationen und magnetischer Domänen, den Nachweis der magnetischen Resonanz einzelner Spins sowie die atomare und molekulare Manipulation von Proben. Unter Bezug auf modernste Forschungsergebnisse werden die genannten Einsatzbereiche der Rasterkraftmikroskopie im Hinblick auf die theoretischen Grundlagen und experimentelle Aspekte detailliert vorgestellt.

Die optische Rasternahfeldmikroskopie erlaubt es, das Beugungslimit der konventionellen Lichtmikroskopie zu durchbrechen und alle konventionellen lichtmikroskopischen Verfahren mit Subwellenlängenauflösung zu realisieren. Daraus resultieren natürlich einige äußerst vielversprechende Anwendungen der optischen Nahfeldmikroskopie, nicht zuletzt auch bei der Charakterisierung biologischer Systeme. Diesbezüglich werden im vorliegenden Band zahlreiche Beispiele diskutiert. Das optische Rasternahfeldmikroskop kann aber nicht nur zur Abbildung sondern auch für die optische Nanolithographie verwendet werden, was bereits zu beeindruckenden Resultaten bei der Herstellung von Nanostrukturen geführt hat.

Das grundlegende Arbeitsprinzip der Rastersondenverfahren lässt sich auf weitere Methoden übertragen, bei denen bei Sonden-Proben-Abständen im Nanometerbereich die Wechselwirkung mit einer Probenoberfläche genutzt wird, um hochaufgelöst abzubilden oder um eine Probe auf Nanometerskala zu manipulieren. Als diesbezügliche Beispiele werden die Ionenleitfähigkeitsmikroskopie, die Thermographie, die SQUID-Mikroskopie und der ungeheuer vielversprechende Bereich der Mikroskopie mit NV-Zentren vorgestellt.

Eine so ausgesprochen umfangreiche und detaillierte Darstellung der Nanostrukturforschung und Nanotechnologie auf der Basis modernster Forschungsergebnisse wäre nicht denkbar, wenn nicht zahlreiche Kolleginnen und Kollegen weltweit ihre Forschungsergebnisse zur Verfügung gestellt hätten. Insbesondere ist die Vielzahl der vorgestellten Ergebnisse aus Grundlagenforschung, angewandter Forschung und Anwendung die Basis dafür, dass das vorliegende Buch wie auch die anderen Bände der Reihe einen ausgesprochen interdisziplinären Charakter besitzt. Ich möchte mich daher bei allen Kolleginnen und Kollegen, die im Zusammenhang mit den entsprechenden Ergebnissen zitiert wurden, explizit für ihre spannenden Ergebnisse bedanken. 
Auch im vorliegenden Fall war für die Bearbeitung oder Herstellung der zahlreichen Abbildungen in diesem Buch Frau Gabriele Kreutzer-Jungmann verantwortlich, bei der ich mich für ihre außerordentlich professionelle Arbeit und für ihr großes Maß an Geduld sehr bedanken möchte. Die Lösung der vielen komplexen Formatierungsprobleme und die Erstellung des druckfertigen Manuskripts lag erneut bei Frau Stefanie Neumann, ohne deren umfangreiche Expertise, große Akribie und erhebliche Geduld die Realisierung dieses Buches in der vorliegenden Form nicht möglich gewesen wäre. Dafür bedanke ich mich herzlich. Zur Reduzierung der Anzahl der Fehler des Buches haben eine Reihe von Personen beigetragen. Für einen ganz erheblichen Beitrag möchte ich an dieser Stelle erneut Herrn Harro Hartmann danken.

Von unschätzbarer Bedeutung für mich war die geduldige und sachkundige Begleitung durch den DeGruyter-Verlag, der die gesamte Buchreihe nun schon seit einigen Jahren betreut. Stellvertretend für das gesamte Team möchte ich hier insbesondere die angenehme Kooperation mit Frau Nadja Schedensack und Frau Kristin Berber-Nerlinger nennen.

Saarbrücken, im April 2019

U. Hartmann 
